\title{
Study of dynamic behavior of grid connected induction generators
}

\author{
K. C. Divya \\ Department of Electrical Engineering \\ Indian Institute of Science \\ Bangalore, India \\ Email:divya@ee.iisc.ernet.in
}

\author{
P. S. Nagendra Rao \\ Department of Electrical Engineering \\ Indian Institute of Science \\ Bangalore, India \\ Email: nagendra@ee.iisc.ernet.in
}

\begin{abstract}
This paper explores the possibility of using a simple model for grid connected induction generators while studying the dynamic behavior of such systems. The approach used here is inspired from the method in use for the transient stability analysis of power systems. In this scheme both the stator and rotor transients of the machines are neglected. This enables the use of torque slip characteristics of the induction machines, in the analysis of their dynamic behavior. A concept of critical clearing slip has been introduced and its utility has been highlighted. The capabilities of the proposed model has been illustrated by studying the behavior of a sample system for the common types of events such as mechanical, electrical and switching disturbances.
\end{abstract}

\section{INTRODUCTION}

Distributed generation (DG) is attracting the attention of utilities as an alternate power generation option. Deregulation, increasing demand, and barriers to building new generation and transmission infrastructure are all contributing to the increased interest in DG. The DGs are sources of electric power that are generally not directly connected to the bulk power transmission system. These are small generation sources located near the load centers. One of the emerging DG application is the wind power. The integration of the increasing component of wind power into the existing distribution infrastructure requires a good understanding of its impact on the grid.

Results of investigation aimed at understanding DG interconnection issues are reported in [1-4]. In all these studies grid connected induction generators driven by wind turbine have been considered. In [1] the stability study has been carried out for a simple system consisting of a single induction generator connected to the infinite bus through a line. This study considers even the network transients. However, considering these transients increases the computational complexity enormously. In [2] and [3] the study has been carried out for a large wind farm, with complete dynamic representation, including the mechanical dynamics of the windmill system. Even though a realistic wind farm has been considered in [2], the induction generators of the entire wind farm have been represented by a single equivalent machine. While in [3] all the machines within the farm are completely represented, the study focuses on analyzing the electromechanical oscillations within a wind farm. In [4] the stability has been analyzed for a wind-hydro hybrid system by linearizing the complete system model around the operating point. However, this can not be used in cases where the disturbance is large, as the linearization may not be valid. The induction generator models used for all these studies is a 5th order model, which considers the stator as well as the rotor transients. Such a model [5] has been extensively used in the past for the study of single induction motors and drives. A very recent work [6] addresses a similar problem in a slightly different context of double fed induction generators. In this the relative performance of the 5th and 3rd order models have been compared, for a single machine stability study.

In the context of distributed generation one is concerned with multi-machine scenarios (interaction of the induction generators with the grid, with other types of generators, etc.). In such a situation the use of a 5th order model to represent the induction machine dynamics poses several problems. The first is that of a large computational effort. This comes about because of the inclusion of the stator and rotor electrical transients, one has to use extremely small integration time steps. Second, even if the individual induction machines are represented by a detailed 5th order model, they have to be interfaced with the network. The manner in which this can be achieved is not very clear. If the transients in the grid are neglected, then there seems to be no justification for including stator transients. If the network transients are included, the problem could become computationally intractable. Moreover, the need for such a detailed representation is yet to be established. Finally, it is not clear whether the inclusion of the exponentially decaying components of electrical transients is desirable. This is in view of the possibility that they could sometimes mask the real phenomena of interest, as they are extremely sensitive to the instant of initiation of the event ('point on wave' issue). Thus, there is a need to seek alternate models for studying the dynamic behavior of practical DG systems that require the representation of several machines.

In this paper, one such alternate approach of analyzing the system dynamics has been investigated. The approach used here, neglects the fast stator and rotor electrical transients. This approximation has been widely used for the transient stability study of system having a number of synchronous machines. This approximation tracks only the slow varying 


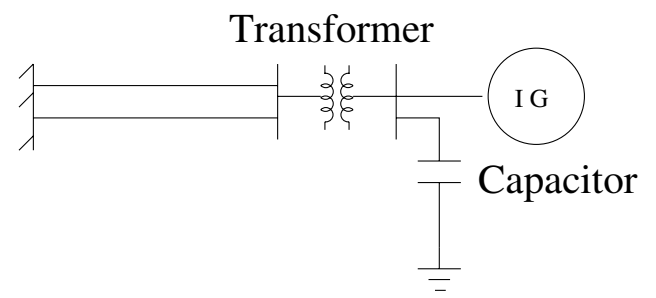

Fig. 1. Test system-one line diagram

dynamics, neglecting the fast decaying electrical transients. This approach seems attractive due to several reasons. It is very simple and computationally not demanding. The utility of this approach is proven in view of its long use in transient stability studies. This scheme facilitates the use of torque-slip characteristics of the induction machines (analogous to the power angle curves used for synchronous machines) to analyze the dynamic behavior of the system.

The assessment of the new model is done by considering a simple test system and studying the impact of changes in mechanical input, short circuit fault, network changes as well as switching disturbances. In this context notion of 'critical clearing slip' is introduced and its utility in these studies is highlighted. The rest of the paper is organized as follows. In the next section the new frame work that is being proposed for the study of the dynamics of induction generators is described. In section III results of simulation studies are presented.

\section{PROPOSED APPROACH}

The proposed method is first explained qualitatively by considering a simple system. The system considered is a single induction generator connected to the infinite bus through a double circuit transmission line as shown in Fig 1.

\section{A. Mechanical disturbances}

In the proposed scheme, all the electrical quantities are obtained from the steady state equivalent circuit of the system. The torque slip characteristic for such a system can be obtained by using the steady state equivalent circuit and is given in Fig 2. Consider the induction machine to be initially operating at a slip S0 as a generator and with the electrical torque, equal to the mechanical input torque Tm0. Now, if the mechanical torque suddenly changes from $\mathrm{Tm} 0$ to $\mathrm{Tmf}$, the rotor experiences a net accelerating torque and the machine accelerates. As the slip increases the electrical torque increases and accelerating torque reduces till a slip Sf (Fig 2) is reached. At this slip the net accelerating power is zero and hence, the machine continues to operate at this slip.

It is seen that the behavior of the induction generator in response to a large step change in mechanical torque is completely different from that of a synchronous machine. A similar disturbance on a synchronous machine would have resulted in the rotor oscillating around $\delta_{f}$ with a large amplitude (a function of $\left|\delta_{0}-\delta_{f}\right|$, where $\delta_{0}$ and $\delta_{f}$ are the initial and final load angles). Where as here the rotor almost monotonically moves from S0 to Sf. There could be a small overshoot at Sf
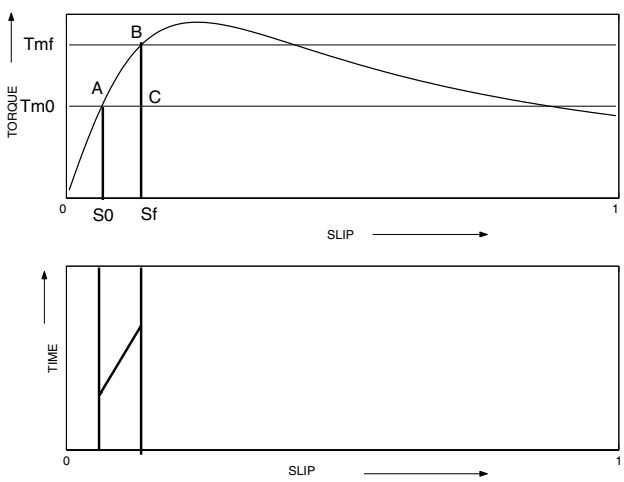

Fig. 2. Torque-slip and time-slip curves for a change in mechanical torque input

depending on $\frac{d \omega}{d t}$ ( $\omega$ is the rotor speed) due to some higher order dynamics that is neglected here. However, in general it will be almost negligible.

For any machine to settle at the new operating point, $\frac{d \omega}{d t}$ at that point must be zero, in addition to the net torque being zero. In a synchronous machine this happens after several oscillation of the rotor with respect to $\delta_{f}$. Where as in the induction generator, this happens the first time the slip reaches Sf. The power mismatch represented by the area ABC in Fig 2 would contribute to the increased kinetic energy of the rotor at the new operating slip Sf. Hence, it is easy to see that in an induction generator a change in the mechanical torque input does not result in any significant mechanical oscillations and the machine does not stall unless the new mechanical input exceeds the break down torque for a sufficiently long duration. Thus, the induction generator seems to simply track the disturbance (with a delay) and it can be said that there is no instability associated with this type of disturbance.

\section{B. Electrical disturbance}

Consider a three phase fault at the induction generator terminals of the sample system. Before the fault the electrical and mechanical torques are equal to $\mathrm{Tm}$ and the machine is operating at slip S0, as shown in Fig 3. Immediately after the fault occurs the electrical torque would be zero (as the electrical transients are ignored), while the slip remains at S0. Thus, there is a net accelerating torque and the slip gradually increases. The fault is cleared at a slip S1, then the electrical torque is assumed to increase instantaneously. However, the slip remains the same. At this operating point the electrical torque is higher than the mechanical torque. Thus, the rotor decelerates (curve-1 of slip-time curve) and settles back to the original slip S0.

Instead of clearing the fault at slip S1, if the fault is cleared at a slip S2, even then the electrical torque increases from zero. However, it will be less than the mechanical torque input and the machine continues to accelerate, which is shown as curve-2 of slip-time curve in Fig 3. Similar behavior can be expected, if the fault is cleared at any point beyond the slip Scr in Fig 3. Thus, the slip Scr can be called as the critical clearing slip for this operating point and disturbance. This implies that if the 

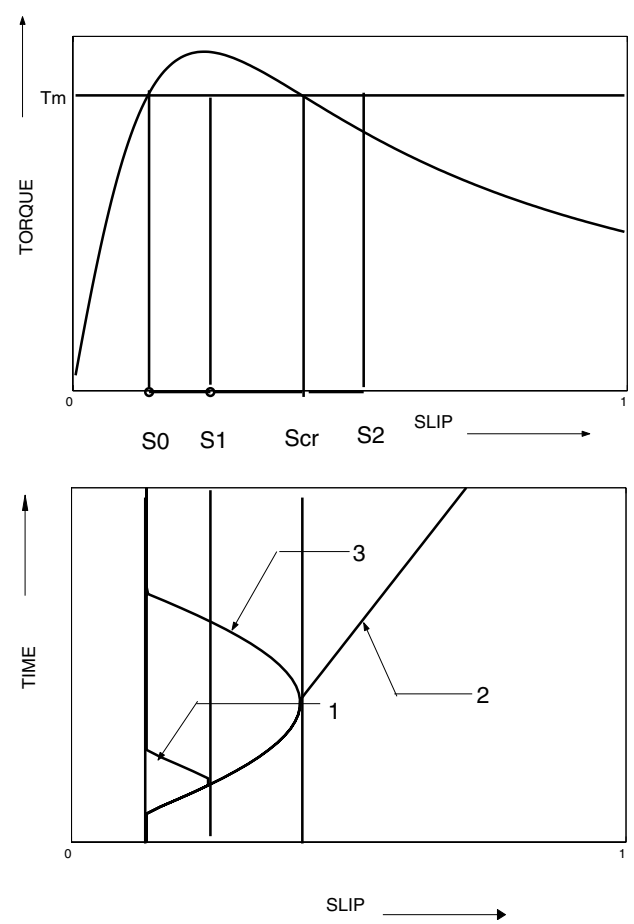

Fig. 3. Torque-slip and time-slip curves for a 3-phase fault

fault is cleared before the slip reaches Scr the machine will not over-speed and get tripped by the speed sensing relays.

The significance of the 'critical clearing slip' is that for a given set of initial operating point and a post fault condition, its value is fixed. It is easy to see that if the initial operating point corresponds to a lower slip, the corresponding critical clearing slip would be much larger (in magnitude). What is more interesting is that the critical clearing slip is independent of the type of fault (conditions during the fault). This implies that, if the system were to experience a different type of fault (say a single line to ground or double line fault) even then, the critical clearing slip will remain unaltered, as long as the pre-fault and post fault conditions remain unchanged. However, the critical clearing time depends on the variation of the accelerating torque of the rotor, which in turn depends on the nature of the fault.

\section{Critical clearing slip/time}

For simple systems as the one considered, an expression for critical clearing slip can be easily derived. The steady state equivalent circuit for the sample system is shown in Fig 4. The algebraic equations corresponding to this can be written as,

$$
V=V_{1}+(R l+\jmath X l+\jmath X t r) I l
$$

where,

$I l=\frac{V_{1}}{-\jmath X c}+I_{1}$ and $V_{1}, I_{1}$ are the stator voltage and current

$$
\begin{aligned}
V_{1} & =(r 1+\jmath X l 1) I_{1}+\jmath X m\left(I_{1}+I_{2}^{\prime}\right) \\
\frac{V_{2}^{\prime}}{s} & =\left(\frac{r 2^{\prime}}{s}+\jmath X l 2^{\prime}\right) I_{2}^{\prime}+\jmath X m\left(I_{1}+I_{2}^{\prime}\right)
\end{aligned}
$$

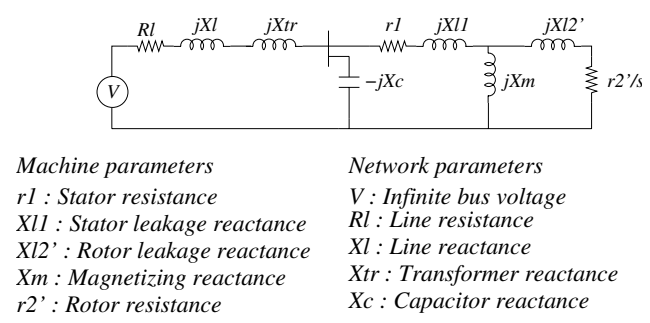

Fig. 4. Equivalent circuit of the sample system

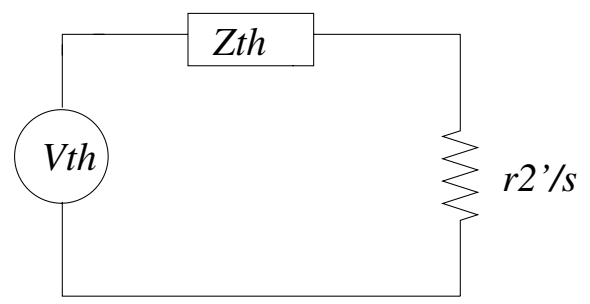

\section{Vth : Thevinin's equivalent voltage}

\section{Zth : Thevinin equivalent impedance}

Fig. 5. Thevinin's equivalent circuit

where,

$V_{2}^{\prime}$ and $I_{2}^{\prime}$ are the rotor voltage and current

The differential equation corresponding to the motion of the rotor is given by,

$$
(2 H) \frac{d s}{d t}=T a(.)=-T m+T e(s)
$$

where,

$T a($.$) : Accelerating torque$

$H$ : inertia constant of the generator in sec

$T m$ : Mechanical torque input

$T e(s)$ : generated electrical torque

$s$ : slip

An analytical expression can be derived for the electrical torque output by considering the Thevinin's equivalent for the circuit shown in Fig 4. The Thevinin's equivalent circuit for this is shown in Fig 5. From this, the electrical torque output of the generator is computed as

$$
T e(s)=\frac{\left|V_{t h}^{2}\right| \frac{r 2^{\prime}}{s}}{\left[\frac{r 2^{\prime}}{s}+R_{t h}\right]^{2}+X_{t h}^{2}}
$$

where,

$Z_{t h}=R_{t h}+\jmath X_{t h}$

The critical clearing slip can be obtained by equating the electrical torque (Te(s) in Eqn 5) to the mechanical input torque and solving it for the slip. For the system considered here, an expression for the critical clearing slip can be obtained as the numerically larger solution of the quadratic equation as

$$
s_{s c r}=-\frac{b}{2 a} \pm \frac{\sqrt{b^{2}-4 a c}}{2 a}
$$

where,

$a=T m\left(R_{t h}^{2}+X_{t h}^{2}\right), b=\left(2 T m R_{t h}-\left|V_{t h}\right|^{2}\right) r 2^{\prime}$ and 
TABLE I

MACHINE AND NETWORK PARAMETERS

\begin{tabular}{|c||c|}
\hline MVA base & 0.674 \\
\hline KV base & 0.69 \\
\hline$r 2^{\prime}$ & $0.0146 \mathrm{pu}$ \\
\hline$r 1$ & $0.0186 \mathrm{pu}$ \\
\hline$X l 1$ & 0.217 \\
\hline$X l 2^{\prime}$ & 0.186 \\
\hline$X m$ & 7.28 \\
\hline$X c$ & 4.17 \\
\hline$X t r$ & 0.0842 \\
\hline$R l$ & 0.000472 \\
\hline$X l$ & 0.0019 \\
\hline
\end{tabular}

$c=\operatorname{Tm}\left(r 2^{\prime}\right)^{2}$

From (6), it is evident that the critical clearing slip is a function of the system parameters and the input mechanical torque, as $V$ remains constant. The system parameters are fixed and the input mechanical torque is assumed to remain constant till the fault is cleared.

One of the applications of critical clearing slip could be in computing the critical clearing time for any fault. This can be done by noting that $-2 H \int_{s_{0}}^{s_{f}} T a(s) d s=\int_{t_{0}}^{t_{f}} T a(t) d t=$ $s_{f}-s_{0}=\Delta s$, if $T a(t)$ is continuous over the interval $t_{0} t_{o} t_{f}$. Then the critical clearing time can be obtained as,

$$
\Delta t=\frac{2 H \Delta s^{2}}{\int_{s_{0}}^{s_{c r}} T a(s) d s}
$$

\section{Simulation STUDY}

Simulation studies have been carried out by considering the single machine system shown in Fig 1. Its parameters are given in Table I. The dynamic behavior of this system has been obtained using the proposed approach for the following cases

1) Step increase in mechanical torque input

2) Three phase fault at the machine terminals

3) Loss of one of the double circuit lines

4) Starting the induction machine from rest

In all the cases, it is assumed that the induction generator is initially supplying an electrical load of $0.8 \mathrm{pu}$, which corresponds to slip of -0.0145 .

\section{A. Mechanical disturbance}

During the normal operation of wind generation system, the wind velocity is randomly varying. This causes a wide variation in the mechanical torque. Thus, it is necessary to study the impact of mechanical torque variation on the induction machine. Hence, the following scenario is considered. The induction machine is initially generating a torque of $0.8 \mathrm{pu}$. At time $\mathrm{t}=2.00 \mathrm{sec}$ the mechanical torque input is increased from 0.8 to $1.0 \mathrm{pu}$.
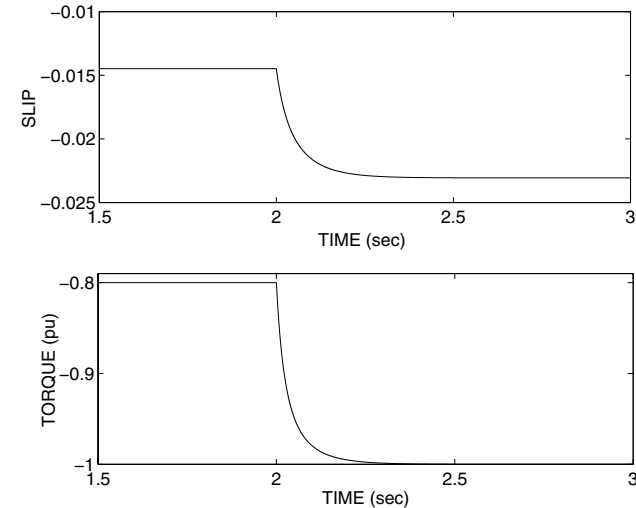

Fig. 6. Torque and slip variation for a step change in the mechanical torque

This is simulated by solving the differential equation (4) and using the electrical torque equation (5), every integration step (step size $=0.01 \mathrm{sec}$ ). For this, the variation in slip and electrical torque are shown in Fig 6. From these plots it can be seen that the electrical torque and the slip gradually settles down to the new value without any oscillation. This is true for any magnitude of change in the mechanical torque, provided the torque input does not exceed the breakdown torque. It is interesting to note that most of the change in speed (slip) occurs immediately after the disturbance. The rate of change of slip falls with time and by the time it reaches the new steady state operating point, the $\frac{d s}{d t}$ is zero. Therefore, for a step change in mechanical torque, the observed dynamic response corresponds to that of a system with a simple first order delay. The settling time for this case can be computed analytical by using (7) and this turns out to be $0.2235 \mathrm{sec}$.

\section{B. Three phase fault}

A three phase short circuit fault at the generator terminals is considered for this study. With such a fault, as the terminal voltage collapses, the computed electrical torque will also be zero. The machine accelerates and will be tripped by speed limit sensors unless the fault is cleared before the machine reaches $S_{c r}$. The time required to reach has been calculated for a three phase fault (at the generator terminals, assuming the pre and post fault conditions to be identical) using the proposed method. The induction generator is initially operating at a slip of $S_{0}=-0.0145$ corresponding to an electrical torque of $T_{e 0}=-0.8 \mathrm{pu}$. The critical clearing slip obtained for this system using (6) is $S_{c r}=-0.0641$. As the accelerating torque is constant during the fault, the critical clearing time can be calculated using (7) as,

$$
t_{c r}=\frac{\left(S_{c r}-S_{0}\right)}{(2 H)\left(T_{e 0}-T m\right)}
$$

The torque-time and slip-time plots for this case are shown in Fig 7. From this it can be seen that, the electrical torque reduces to zero at the instant of the three phase fault $(t=2.00 \mathrm{sec})$. While the slip (rotor speed) gradually increases, till the fault is cleared $(t=2.0589 \mathrm{sec})$. As soon as the fault is cleared, the electrical torque increases to $0.8 \mathrm{pu}$. The slip gradually 

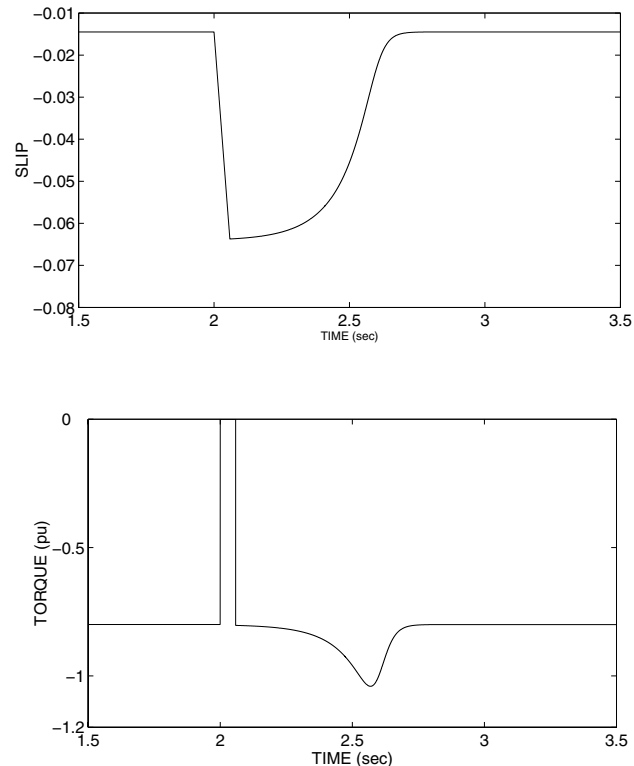

Fig. 7. Torque and slip variation for a three phase fault
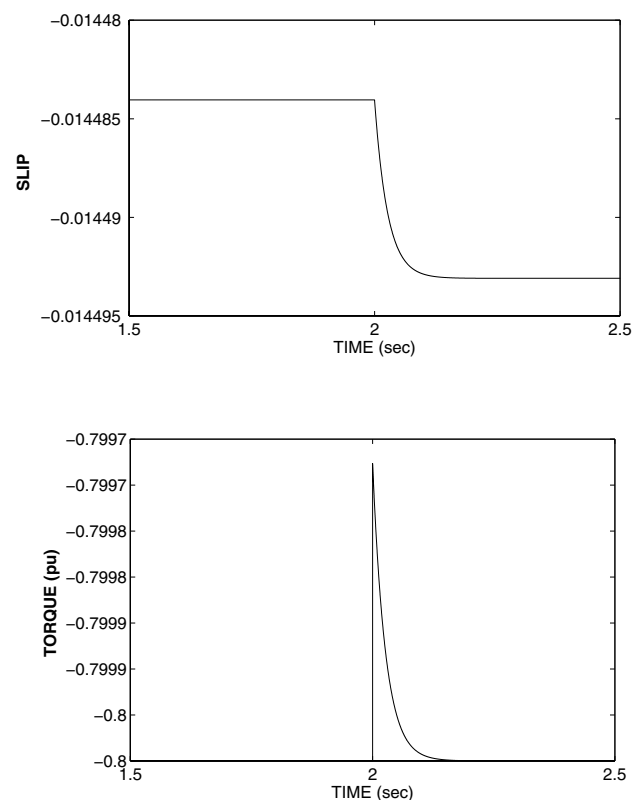

Fig. 8. Torque and slip variation : Opening one line of a double circuit transmission line

decreases and takes a very long time to settle down, as compared to the fault duration. An overshoot is also seen in the electrical torque before the machine settles down at the original operating point.

\section{Network changes}

The most common network change that occurs in a actual system is due to the opening of one of the lines of a double circuit transmission line. This has been studied here by considering the sample system. One of the lines of this system is opened at $\mathrm{t}=2.00 \mathrm{sec}$, the variation in torque and slip are shown in Fig 8. From the plots it can be seen that the electrical
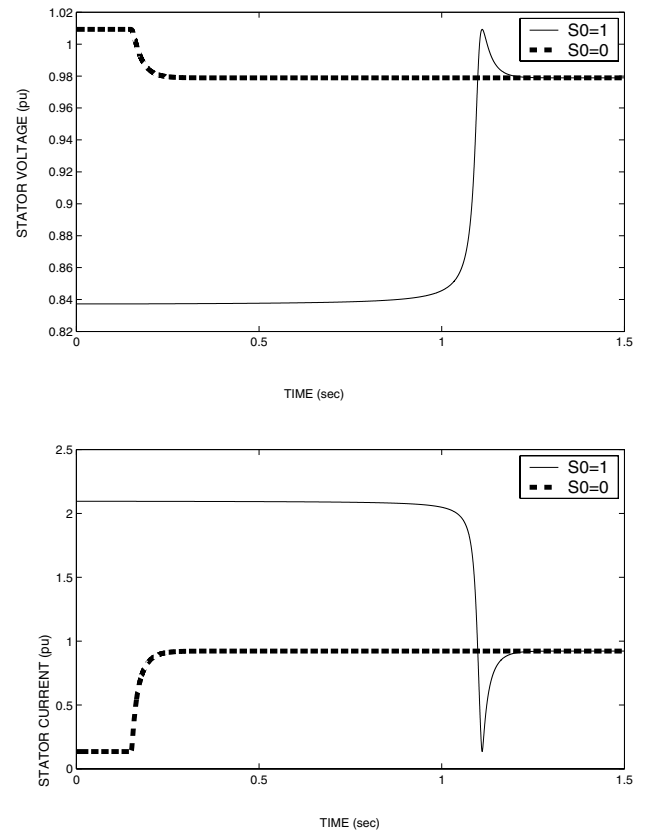

Fig. 9. Variation in stator voltage and current during switching

torque changes instantaneously by a very small amount, while the slip remains the same. The instantaneous fall in electrical torque is due to the sudden fall in stator voltage caused by the opening of one of the lines. This change in the electrical torque disturbs the torque equilibrium resulting in a gradual increase in the magnitude of the slip. The electrical torque restores back to its original value $(-0.8 \mathrm{pu})$ when the slip increases to -0.014495 , a marginally higher value. It is interesting to note that a major disturbance such as a loss of line does not cause any significant disturbance. A similar situation in the case of a synchronous machine could lead to a serious stability problem.

\section{Starting transients}

The wind generation system is switched on to the grid, when the wind velocity reaches a cut in speed. They can be synchronized with the grid in many ways. The extremes being, switching on to the grid when the induction machines are at rest or are operating near synchronous speed. In all the cases, this switching in process causes a lagging current to be drawn initially from the grid there by causing a dip in the voltage at the stator terminals. Thus it is necessary to know the magnitude of increase in the stator current as well as the magnitude of voltage dip, in these two extreme cases. The effect of connecting the induction machine to the grid when at rest and when running near synchronous speed has been studied for the sample system (Fig 1).

This simulation has been carried out as follows. For both the cases the simulation is started at $\mathrm{t}=0.0 \mathrm{sec}$ with the rated voltage applied. In one case the slip at $\mathrm{t}=0$ is taken as zero $(\mathrm{S} 0=0)$ and for the other case it is taken as $1(\mathrm{~S} 0=1)$. The mechanical torque is assumed to be applied after a small delay $(t=0.15 \mathrm{sec})$ in both the cases. The variation in stator voltage and current for these two cases are shown in Fig 9. From 
Fig 9 (considering the case when the machine is started with a slip $\mathrm{S}(=0)$ it is seen that when the mechanical torque is applied the stator current magnitude increases for about 0.1 sec and reaches a final value of $0.9107 \mathrm{pu}$. The voltage falls marginally, as the reactive demand of the machine increases. In the other case (the machine is started with slip $\mathrm{S} 0=1$ ) at starting, a very large voltage dip of about $16.5 \%$ is seen. This persists for about one second. In this period, current remains very high (around $2 \mathrm{pu}$ ). After about a second as the machine changes from motoring to generating mode, we observe a sudden current dip which subsequently rises to $0.9107 \mathrm{pu}$. This sudden dip in the current reflects as a small voltage spike at about $1.1 \mathrm{sec}$. It must be however pointed out that if the machine were to be started as an induction motor (without applying mechanical torque at $0.15 \mathrm{sec}$ ) it would take about $7 \mathrm{sec}$ to settle down to its no load speed. Consequently, the voltage dip at the machine terminal would persist for a very long time. This suggests that applying mechanical input to the induction generator at starting would help in reducing the starting transient to a great extent.

\section{CONCLUSION}

This paper is concerned with the analysis of the dynamics of DG system having many induction generators. It is argued that simple models for representing induction generators are needed, especially when analyzing such systems. One simple model has been examined in detail. The use of such simple models to the study the dynamic behavior of a test system (single induction generator connected to the infinite bus through a line) has been analyzed. The dynamic behavior of the system has been studied for the most common types of disturbances. For these disturbances, it is shown that the dynamic behavior of the induction generator is significantly different from that of the synchronous machine.

This study clearly brings out the types of the dynamic behavior that can be obtained through simulation using the proposed simple representation. It is true that the dynamics obtained by this model could be significantly different from the ones obtained using detailed models. However, the feasibility as well as the need of using such detailed models, from a practical point of view is still an open issue. The conjecture here is that the simple model proposed here could be adequate in many situations. Efforts are continuing to understand the issues so that one may be able to answer these questions in a more concrete manner.

\section{REFERENCES}

[1] Senjyu T., Sueyoshi N., Uezato K. and Fujita H., "Stability analysis of wind power generating system", Proceedings of the Power Conversion Conference-Osaka 2002, 2-5 April 2002, vol. 3, no. 3, pp. 1441-6.

[2] Akhmatov V., Knudsen H., Nielsen A.H., Pedersen, J.K. and Poulsen, N.K., "An aggregate model of a grid-connected, large scale, offshore wind farm for power stability investigations-importance of windmill mechanical system", International Journal of Electrical Power and Energy Systems, 2002, vol. 24, no. 9, pp. 709-717.

[3] Akhmatov V., Knudsen H., Nielsen A.H., Pedersen J.K. and Poulsen N.K., "Modelling and transient stability of large wind farms", International Journal of Electrical Power and Energy Systems, 2003, vol. 25, no. 2, pp. 123-44.
[4] Fan Yanfang, Wang Yibo and Chao Qin, "Wind-hydro hybrid power system stability analysis", Proceedings of the Fifth International Conference on Electrical Machines and Systems, 18-20 Aug. 2001, vol. 1, no. 1, pp. 628-32.

[5] Krause P. C., Analysis of electric machinery, McGraw-Hill, 1986.

[6] Ekanayake J. B., Holdsworth L. and Jenkins N., "Comparison of the 5th and 3rd order machine models for doubly fed induction generator (DFIG) wind turbines", Electric Power Systems Research, 2003, vol. 67, pp. $207-$ 215. 